\section{Review: certain types of developmental care result in some benefits for preterm infants}

Symington A, Pinelli J. Developmental care for promoting development and preventing morbidity in preterm infants.

Cochrane Database Syst Rev 2000;(4):CD001814 (latest version 28 July 2000). QUESTION: Does developmental care (ie, interventions designed to modify the
neonatal intensive care unit [NICU] environment and thereby minimise the stress
experienced by preterm infants) reduce adverse outcomes in preterm infants?
Source of funding: no external funding

For correspondence: Ms A Symington, Acute Children's Services, Hamilton Health Sciences Corporation, $P O$ Box 2000, Hamiltom, Ontario L8N $3 Z 5$, Canada.Fax +1905 5215007.

\section{Data sources}

Studies were identified by searching Medline (1966 to July 2000), CINAHL, and the Cochrane Library; English language conference and symposia proceedings from 1990 to July 2000; bibliographies of retrieved articles; and by contacting experts.

\section{Study selection}

Randomised trials were included if they compared elements of developmental care with routine nursery care in infants $<37$ weeks gestation. Interventions of interest were positioning, clustering of nursery care activities, modification of external stimuli, and individualised developmental care interventions.

\section{Data extraction}

Data were extracted on methods, participants, interventions, and outcomes. The authors independently assessed the methodological quality of studies.

\section{Main results}

31 trials $(n=2009)$ met the inclusion criteria. The table summarises the results.

\section{Conclusion}

Developmental care for preterm infants shows some evidence of benefit for improved short term growth and neurodevelopmental outcomes to 24 months corrected age, and decreased respiratory support and length and cost of hospital stay.

\section{COMMENTARY}

Preterm infants may be adversely affected by the sensory environment of the NICU. Developmental care was introduced in the mid- $1980 \mathrm{~s}^{1}$ to minimise the stress experienced by preterm infants through modification of the environment using such strategies as noise and light reduction and minimal handling. Although developmental care has been widely accepted in NICUs in North America and abroad, little consensus exists about the benefits of individual components of developmental care.

This systematic review by Symington and Pinelli concludes that developmental care provides benefits in the form of improved short term growth and neurodevelopmental outcomes, and decreased respiratory support and length of hospital stay. These findings, however, are based on 2 or 3 small trials for each outcome. Although other benefits were found, these were based on single studies with small sample sizes. For many outcomes, no or conflicting effects were found. Of the 31 studies included in the review, 9 had adequate allocation concealment, 15 had blinded outcome measurement, and 23 had complete follow up of study participants. No harmful effects of developmental care were found, and none of the studies considered the cost of developmental care.

NICU staff nurses, clinical nurse specialists, neonatal nurse practitioners, and other developmental care team members will find the results of this systematic review interesting, especially those in those units where developmental care is implemented. Symington and Pinelli conclude that the jury is still out and that evidence of more consistent effects of developmental care interventions on important short and long term clinical outcomes is needed. They recommend that the economic effects of the implementation and maintenance of developmental care practices should be considered by individual institutions. Debbie Fraser Askin, RNC, MN Neonatal Nurse Practitioner Assistant Professor, Faculty of Nursing University of Manitoba Winnipeg, Manitoba, Canada

1 Als $\mathrm{H}$, Lawhon G, Brown E, et al. Individualized behavioral and environmental care for the very low birth weight preterm infant at high risk for bronchopulmonary dysplasia: neonatal intensive care unit and developmental outcome. Pediatrics 1986;78:1123-32.

Developmental care $v$ control for preterm infants

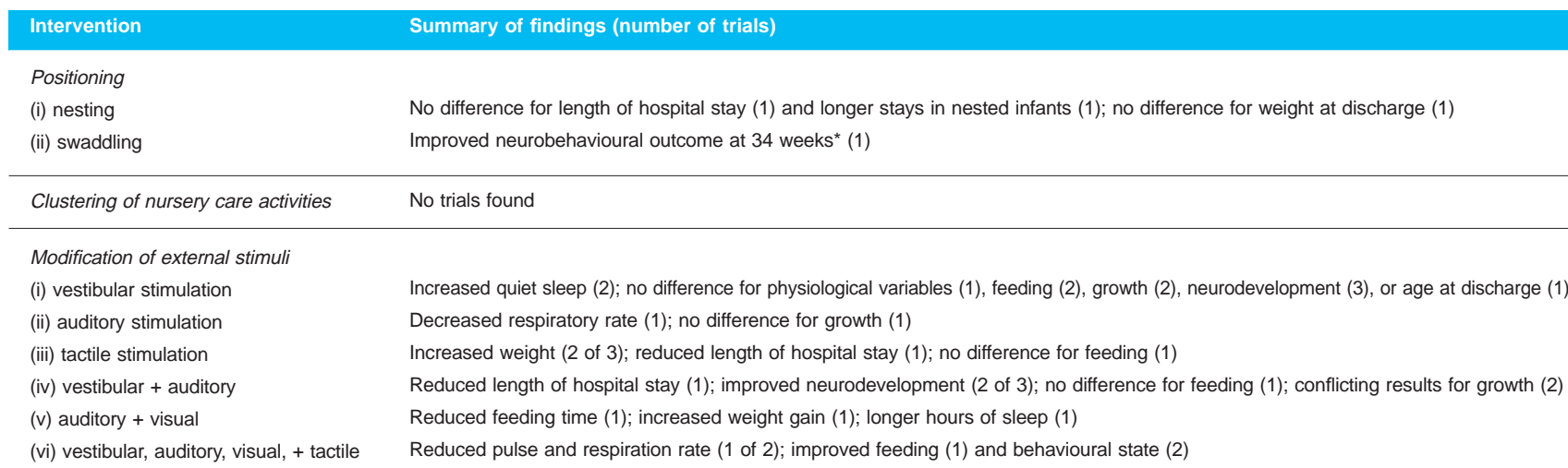

Individualised developmental care

(i) Neonatal Individualized Developmental Care and Assessment Program

(ii) other individualised interventions
Decreased ventilation days (2†), tube feeding days (2†), gestational age at discharge (2†), length of hospital stay (2†), and hospital charges (1); improved neurodevelopment at 9 months (1 of 2); no difference for rate of pneumothorax (2†), patent ductus arteriosis (1), chronic lung disease $(2 \dagger)$, intraventricular haemorrhage $(2 \dagger)$, retinopathy of prematurity (2), sepsis (1), weight gain (3), death before discharge (1), or neurodevelopment at 12 months (1).

Decreased ventilation days (1); improved neurodevelopment at 12 but not 24 months(1); no differences for neonatal outcomes (1), feeding and growth (2), and length (2†) and cost of hospital stay (1) 